Bull. Korean Math. Soc. 48 (2011), No. 4, pp. 697-704

DOI 10.4134/BKMS.2011.48.4.697

\title{
ON SUFFICIENT CONDITIONS FOR STRONGLY STARLIKE FUNCTIONS ASSOCIATED WITH A LINEAR OPERATOR
}

\author{
JIN-LIN LIU
}

ABSTRACT. By using the method of differential subordinations, we derive some sufficient conditions for strongly starlike functions associated with a linear operator. All these results presented here are sharp.

\section{Introduction and preliminaries}

Let $A_{p}$ denote the class of functions of the form

$$
f(z)=z^{p}+\sum_{n=1}^{\infty} a_{n} z^{n+p} \quad(p \in \mathbb{N}=\{1,2,3, \ldots\}),
$$

which are analytic in the open unit disk $U=\{z: z \in \mathbb{C}$ and $|z|<1\}$. A function $f(z) \in A_{p}$ is called $p$-valent starlike in $U$ if it satisfies

$$
\operatorname{Re} \frac{z f^{\prime}(z)}{f(z)}>0 \quad(z \in U) .
$$

Also, a function $f(z) \in A_{p}$ is called $p$-valent strongly starlike of order $\alpha(0<$ $\alpha \leq 1)$ if it satisfies

$$
\left|\arg \left(\frac{z f^{\prime}(z)}{f(z)}\right)\right|<\frac{\pi}{2} \alpha \quad(z \in U) .
$$

For functions $f_{j}(z) \in A_{p}(j=1,2)$ given by

$$
f_{j}(z)=z^{p}+\sum_{n=1}^{\infty} a_{n, j} z^{n+p}(j=1,2),
$$

we define the Hadamard product (or convolution) of $f_{1}(z)$ and $f_{2}(z)$ by

$$
\left(f_{1} * f_{2}\right)(z)=z^{p}+\sum_{n=1}^{\infty} a_{n, 1} a_{n, 2} z^{n+p}=\left(f_{2} * f_{1}\right)(z) .
$$

Received August 14, 2009; Revised March 3, 2010

2010 Mathematics Subject Classification. Primary 30C45; Secondary 30A10.

Key words and phrases. analytic function, starlike function, strongly starlike function, subordination, Hadamard product (or convolution). 
Suppose that $f(z)$ and $g(z)$ are analytic in $U$. We say that the function $f(z)$ is subordinate to $g(z)$ in $U$, and we write $f(z) \prec g(z)$, if there exists an analytic function $w(z)$ in $U$ with $w(0)=0$ and $|w(z)|<1(z \in U)$, such that $f(z)=g(w(z))(z \in U)$. If $g(z)$ is univalent in $U$, then the following equivalence relationship holds true.

$$
f(z) \prec g(z)(z \in U) \Longleftrightarrow f(0)=g(0) \text { and } f(U) \subset g(U) .
$$

For a function $f(z) \in A_{p}$, we consider a linear operator $Q_{p, \lambda_{2}}^{\lambda_{1}}: A_{p} \rightarrow A_{p}$ as following:

$$
\begin{aligned}
Q_{p, \lambda_{2}}^{\lambda_{1}} f(z)= & \left(\begin{array}{c}
p+\lambda_{1}+\lambda_{2}-1 \\
p+\lambda_{2}-1
\end{array}\right) \frac{\lambda_{1}}{z^{\lambda_{2}}} \int_{0}^{z}\left(1-\frac{t}{z}\right)^{\lambda_{1}-1} t^{\lambda_{2}-1} f(t) d t \\
& \left(\lambda_{1}>0, \lambda_{2}>-1 \quad \text { and } \quad f(z) \in A_{p}\right) .
\end{aligned}
$$

We note that

$$
\begin{gathered}
Q_{p, \lambda_{2}}^{\lambda_{1}} f(z)=z^{p}+\sum_{n=1}^{\infty} \frac{\Gamma\left(p+n+\lambda_{2}\right) \Gamma\left(p+\lambda_{1}+\lambda_{2}\right)}{\Gamma\left(p+n+\lambda_{1}+\lambda_{2}\right) \Gamma\left(p+\lambda_{2}\right)} a_{p+n} z^{p+n} \\
\left(\lambda_{1}>0, \lambda_{2}>-1 \quad \text { and } \quad f(z) \in A_{p}\right) .
\end{gathered}
$$

In particular, we have

$$
Q_{p, \lambda_{2}}^{0} f(z)=f(z) \text { for } \lambda_{1}=0 \text { and } f(z) \in A_{p} .
$$

The operator $Q_{p, \lambda_{2}}^{\lambda_{1}}$ was introduced by Liu and Owa [4]. When $p=1$, the operator $Q_{p, \lambda_{2}}^{\lambda_{1}}$ was first introduced by Jung et al. [2]. Many interesting subclasses of analytic functions, associated with the operator $Q_{p, \lambda_{2}}^{\lambda_{1}}$, have been considered by Jung et al. [2], Aouf et al. [1], Liu [3], Liu and Owa [4] and others.

In order to prove our main results, we need the following lemma.

Lemma. Let the function $g(z)$ be analytic and univalent in $U$ and let the functions $\theta(w)$ and $\varphi(w)$ be analytic in a domain $D$ containing $g(U)$, with $\varphi(w) \neq 0(w \in g(U))$. Set

$$
Q(z)=z g^{\prime}(z) \varphi(g(z)) \quad \text { and } \quad h(z)=\theta(g(z))+Q(z)
$$

and suppose that

(i) $Q(z)$ is univalently starlike in $U$ and

(ii) $\operatorname{Re} \frac{z h^{\prime}(z)}{Q(z)}=\operatorname{Re}\left(\frac{\theta^{\prime}(g(z))}{\varphi(g(z))}+\frac{z Q^{\prime}(z)}{Q(z)}\right)>0 \quad(z \in U)$.

If $q(z)$ is analytic in $U$ with $q(0)=g(0), q(U) \subset D$ and

$$
\theta(q(z))+z q^{\prime}(z) \varphi(q(z)) \prec \theta(g(z))+z g^{\prime}(z) \varphi(g(z))=h(z) \quad(z \in U),
$$

then $q(z) \prec g(z)(z \in U)$ and $g(z)$ is the best dominant of (1.5).

The lemma is due to Miller and Mocanu [5, p. 132]. 


\section{Sufficient conditions for strongly starlike functions}

In this section, we assume that $\alpha, \lambda_{0}, \lambda, a, b \in \mathbb{R}$ and $\mu \in \mathbb{C}$.

Theorem 1. Let

$$
0<\alpha \leq 1, \lambda_{0} a \geq 0,|b+1| \leq \frac{1}{\alpha} \quad \text { and } \quad|a-b-1| \leq \frac{1}{\alpha} .
$$

If $f(z) \in A_{p}$ satisfies $Q_{p, \lambda_{2}}^{\lambda_{1}} f(z)\left(Q_{p, \lambda_{2}}^{\lambda_{1}} f(z)\right)^{\prime} \neq 0(z \in U \backslash\{0\})$ and

$\lambda_{0}\left(\frac{z\left(Q_{p, \lambda_{2}}^{\lambda_{1}} f(z)\right)^{\prime}}{p Q_{p, \lambda_{2}}^{\lambda_{1}} f(z)}\right)^{a}+z\left(\frac{z\left(Q_{p, \lambda_{2}}^{\lambda_{1}} f(z)\right)^{\prime}}{p Q_{p, \lambda_{2}}^{\lambda_{1}} f(z)}\right)^{\prime}\left(\frac{z\left(Q_{p, \lambda_{2}}^{\lambda_{1}} f(z)\right)^{\prime}}{p Q_{p, \lambda_{2}}^{\lambda_{1}} f(z)}\right)^{b} \prec h(z)(z \in U)$,

where

$$
h(z)=\lambda_{0}\left(\frac{1+z}{1-z}\right)^{a \alpha}+\left(\frac{1+z}{1-z}\right)^{(b+1) \alpha} \cdot \frac{2 \alpha z}{1-z^{2}},
$$

then the function $Q_{p, \lambda_{2}}^{\lambda_{1}} f(z)$ is p-valent strongly starlike of order $\alpha$ in $U$. The number $\alpha$ is sharp for the function $f(z)$ defined by

$$
\frac{z\left(Q_{p, \lambda_{2}}^{\lambda_{1}} f(z)\right)^{\prime}}{p Q_{p, \lambda_{2}}^{\lambda_{1}} f(z)}=\left(\frac{1+z}{1-z}\right)^{\alpha} .
$$

Proof. We choose

$$
q(z)=\frac{z\left(Q_{p, \lambda_{2}}^{\lambda_{1}} f(z)\right)^{\prime}}{p Q_{p, \lambda_{2}}^{\lambda_{1}} f(z)}, \quad g(z)=\left(\frac{1+z}{1-z}\right)^{\alpha}, \quad \theta(w)=\lambda_{0} w^{a} \quad \text { and } \quad \varphi(w)=w^{b}
$$

in Lemma. Clearly, the function $g(z)$ is analytic and univalently convex in $U$ and

$$
|\arg g(z)|<\frac{\pi}{2} \alpha \leq \frac{\pi}{2} \quad(z \in U)
$$

The function $q(z)$ is analytic in $U$ with $q(0)=g(0)=1$ and $q(z) \neq 0 \quad(z \in U)$. The functions $\theta(w)$ and $\varphi(w)$ are analytic in a domain $D$ containing $g(U)$ and $q(U)$, with $\varphi(w) \neq 0$ when $w \in g(U)$. For

the function $Q(z)$ given by

$$
-\frac{1}{\alpha} \leq b+1 \leq \frac{1}{\alpha}
$$

$$
Q(z)=z g^{\prime}(z) \varphi(g(z))=\frac{2 \alpha z}{(1-z)^{1+(b+1) \alpha}(1+z)^{1-(b+1) \alpha}}
$$

is univalently starlike in $U$ because

$$
\begin{aligned}
\operatorname{Re} \frac{z Q^{\prime}(z)}{Q(z)} & =1+(1+(b+1) \alpha) \operatorname{Re} \frac{z}{1-z}-(1-(b+1) \alpha) \operatorname{Re} \frac{z}{1+z} \\
& >1-\frac{1}{2}(1+(b+1) \alpha)-\frac{1}{2}(1-(b+1) \alpha)=0 \quad(z \in U) .
\end{aligned}
$$


Further, we have

$$
\begin{aligned}
\theta(g(z))+Q(z) & =\lambda_{0}\left(\frac{1+z}{1-z}\right)^{a \alpha}+\frac{2 \alpha z}{(1-z)^{1+(b+1) \alpha}(1+z)^{1-(b+1) \alpha}} \\
& =h(z),
\end{aligned}
$$

where $h(z)$ is given by $(2.3)$, and so

$$
\begin{aligned}
\frac{z h^{\prime}(z)}{Q(z)} & =\frac{\theta^{\prime}(g(z))}{\varphi(g(z))}+\frac{z Q^{\prime}(z)}{Q(z)} \\
& =\lambda_{0} a(g(z))^{a-b-1}+\frac{z Q^{\prime}(z)}{Q(z)} .
\end{aligned}
$$

Also, for

we find that

$$
|a-b-1| \leq \frac{1}{\alpha}
$$

$$
\left|\arg (g(z))^{a-b-1}\right| \leq|a-b-1| \cdot \frac{\alpha \pi}{2} \leq \frac{\pi}{2} \quad(z \in U) .
$$

Therefore, it follows from (2.1) and (2.5) to (2.8) that

$$
\operatorname{Re} \frac{z h^{\prime}(z)}{Q(z)}>0 \quad(z \in U) .
$$

The other conditions of Lemma are also satisfied. Hence we conclude that

$$
q(z)=\frac{z\left(Q_{p, \lambda_{2}}^{\lambda_{1}} f(z)\right)^{\prime}}{p Q_{p, \lambda_{2}}^{\lambda_{1}} f(z)} \prec\left(\frac{1+z}{1-z}\right)^{\alpha}=g(z) \quad(z \in U)
$$

and $g(z)$ is the best dominant of (2.2). By (2.5) we see that the function $Q_{p, \lambda_{2}}^{\lambda_{1}} f(z)$ is $p$-valent strongly starlike of order $\alpha$ in $U$.

Furthermore, for the function $f(z)$ defined by (2.4), we have

$$
\lambda_{0}(q(z))^{a}+z q^{\prime}(z)(q(z))^{b}=h(z),
$$

which shows that the number $\alpha$ is sharp. The proof of Theorem 1 is now completed.

Theorem 2. Let

$$
0<\alpha \leq 1, \lambda(b+2) \geq 0,(b+1) \operatorname{Re} \mu \geq 0 \quad \text { and } \quad|b+1| \leq \frac{1}{\alpha} .
$$

If $f(z) \in A_{p}$ satisfies $Q_{p, \lambda_{2}}^{\lambda_{1}} f(z)\left(Q_{p, \lambda_{2}}^{\lambda_{1}} f(z)\right)^{\prime} \neq 0(z \in U \backslash\{0\})$ and

$$
\begin{aligned}
& \lambda\left(\frac{z\left(Q_{p, \lambda_{2}}^{\lambda_{1}} f(z)\right)^{\prime}}{p Q_{p, \lambda_{2}}^{\lambda_{1}} f(z)}\right)^{b+2}+\mu\left(\frac{z\left(Q_{p, \lambda_{2}}^{\lambda_{1}} f(z)\right)^{\prime}}{p Q_{p, \lambda_{2}}^{\lambda_{1}} f(z)}\right)^{b+1} \\
& +z\left(\frac{z\left(Q_{p, \lambda_{2}}^{\lambda_{1}} f(z)\right)^{\prime}}{p Q_{p, \lambda_{2}}^{\lambda_{1}} f(z)}\right)^{\prime}\left(\frac{z\left(Q_{p, \lambda_{2}}^{\lambda_{1}} f(z)\right)^{\prime}}{p Q_{p, \lambda_{2}}^{\lambda_{1}} f(z)}\right)^{b} \prec h(z) \quad(z \in U),
\end{aligned}
$$


where

$$
h(z)=\left(\frac{1+z}{1-z}\right)^{(b+1) \alpha}\left(\mu+\lambda\left(\frac{1+z}{1-z}\right)^{\alpha}+\frac{2 \alpha z}{1-z^{2}}\right),
$$

then the function $Q_{p, \lambda_{2}}^{\lambda_{1}} f(z)$ is p-valent strongly starlike of order $\alpha$ in $U$. The number $\alpha$ is sharp for the function $f(z)$ defined by (2.4).

Proof. Let

$q(z)=\frac{z\left(Q_{p, \lambda_{2}}^{\lambda_{1}} f(z)\right)^{\prime}}{p Q_{p, \lambda_{2}}^{\lambda_{1}} f(z)}, g(z)=\left(\frac{1+z}{1-z}\right)^{\alpha}, \theta(w)=\lambda w^{b+2}+\mu w^{b+1}, \varphi(w)=w^{b}$

in Lemma. Clearly, the functions $q(z), g(z), \theta(w), \varphi(w)$ and $Q(z)=z g^{\prime}(z) \varphi(g(z))$ satisfy the conditions of Lemma respectively. Further, we have

$$
\begin{aligned}
\theta(g(z))+Q(z)= & \lambda\left(\frac{1+z}{1-z}\right)^{(b+2) \alpha}+\mu\left(\frac{1+z}{1-z}\right)^{(b+1) \alpha} \\
& +\frac{2 \alpha z}{(1-z)^{1+(b+1) \alpha}(1+z)^{1-(b+1) \alpha}} \\
= & h(z)
\end{aligned}
$$

where $h(z)$ is given by $(2.11)$, and so

$$
\begin{aligned}
\frac{z h^{\prime}(z)}{Q(z)} & =\frac{\theta^{\prime}(g(z))}{\varphi(g(z))}+\frac{z Q^{\prime}(z)}{Q(z)} \\
& =\lambda(b+2) g(z)+\mu(b+1)+\frac{z Q^{\prime}(z)}{Q(z)} .
\end{aligned}
$$

Now, for

$$
\lambda(b+2) \geq 0 \quad \text { and } \quad(b+1) \operatorname{Re} \mu \geq 0,
$$

we have

$$
\operatorname{Re} \frac{z h^{\prime}(z)}{Q(z)}>0 \quad(z \in U) .
$$

The other conditions of Lemma are also satisfied. Hence we obtain the desired result of the theorem.

Furthermore, for the function $f(z)$ defined by (2.4), we have

$$
\lambda(q(z))^{b+2}+\mu(q(z))^{b+1}+z q^{\prime}(z)(q(z))^{b}=h(z),
$$

which shows that the number $\alpha$ is sharp. The proof of Theorem 2 is completed.

Theorem 3. Let

$$
0<\alpha \leq 1, \quad \mu>0 \quad \text { and } \quad 0 \leq(b+1) \alpha \leq 1 .
$$


If $f(z) \in A_{p}$ satisfies $Q_{p, \lambda_{2}}^{\lambda_{1}} f(z)\left(Q_{p, \lambda_{2}}^{\lambda_{1}} f(z)\right)^{\prime} \neq 0(z \in U \backslash\{0\})$ and for $z \in U$ $(2.13)$

$\left|\arg \left\{\mu\left(\frac{z\left(Q_{p, \lambda_{2}}^{\lambda_{1}} f(z)\right)^{\prime}}{p Q_{p, \lambda_{2}}^{\lambda_{1}} f(z)}\right)^{b+1}+z\left(\frac{z\left(Q_{p, \lambda_{2}}^{\lambda_{1}} f(z)\right)^{\prime}}{p Q_{p, \lambda_{2}}^{\lambda_{1}} f(z)}\right)^{\prime}\left(\frac{z\left(Q_{p, \lambda_{2}}^{\lambda_{1}} f(z)\right)^{\prime}}{p Q_{p, \lambda_{2}}^{\lambda_{1}} f(z)}\right)^{b}\right\}\right|<\frac{\pi}{2} \beta$,

where

$$
\beta=(b+1) \alpha+\frac{2}{\pi} \tan ^{-1}\left(\frac{\alpha}{\mu}\right),
$$

then

$$
\left|\arg \left(\frac{z\left(Q_{p, \lambda_{2}}^{\lambda_{1}} f(z)\right)^{\prime}}{Q_{p, \lambda_{2}}^{\lambda_{1}} f(z)}\right)\right|<\frac{\pi}{2} \alpha \quad(z \in U) .
$$

This shows that the function $Q_{p, \lambda_{2}}^{\lambda_{1}} f(z)$ is p-valent strongly starlike of order $\alpha$ in $U$. The bound $\beta$ in (2.13) is the largest number such that (2.15) holds true.

Proof. By taking

$$
\lambda=0, \quad \mu>0 \quad \text { and } \quad 0 \leq b+1 \leq \frac{1}{\alpha}
$$

in Theorem 2, we see that if

$$
\mu\left(\frac{z\left(Q_{p, \lambda_{2}}^{\lambda_{1}} f(z)\right)^{\prime}}{p Q_{p, \lambda_{2}}^{\lambda_{1}} f(z)}\right)^{b+1}+z\left(\frac{z\left(Q_{p, \lambda_{2}}^{\lambda_{1}} f(z)\right)^{\prime}}{p Q_{p, \lambda_{2}}^{\lambda_{2}} f(z)}\right)^{\prime}\left(\frac{z\left(Q_{p, \lambda_{2}}^{\lambda_{1}} f(z)\right)^{\prime}}{p Q_{p, \lambda_{2}}^{\lambda_{1}} f(z)}\right)^{b} \prec h(z), z \in U,
$$

where

$$
h(z)=\left(\frac{1+z}{1-z}\right)^{(b+1) \alpha}\left(\mu+\frac{2 \alpha z}{1-z^{2}}\right),
$$

then (2.15) is true.

For $z=e^{i \theta}(\theta \in \mathbb{R}), z \neq 1$ and $z \neq-1$, we get

$$
\begin{gathered}
\frac{z}{1-z}=-\frac{1}{2}+\frac{i}{2} \cot \frac{\theta}{2}, \quad \frac{z}{1+z}=\frac{1}{2}+\frac{i}{2} \tan \frac{\theta}{2}, \\
\frac{1+z}{1-z}=\frac{1+e^{i \theta}}{1-e^{i \theta}}=\cot \frac{\theta}{2} e^{\frac{\pi}{2} i} \neq 0 .
\end{gathered}
$$

The following two cases arise.

(i) If

$$
k(\theta)=\cos \frac{\theta}{2} \sin \frac{\theta}{2}=\frac{1}{2} \sin \theta>0,
$$

then we deduce from (2.17) to (2.19) that

$$
h\left(e^{i \theta}\right)=\left(\cot \frac{\theta}{2}\right)^{(b+1) \alpha} e^{\frac{1}{2}(b+1) \alpha \pi i}\left(\mu+i \frac{\alpha}{2}\left(\cot \frac{\theta}{2}+\tan \frac{\theta}{2}\right)\right),
$$


which yields

$$
\operatorname{argh}\left(e^{i \theta}\right)=\frac{1}{2}(b+1) \alpha \pi+\tan ^{-1}\left(\frac{\alpha}{2 \mu k(\theta)}\right)
$$

for $\mu>0, e^{i \theta} \neq 1$ and $e^{i \theta} \neq-1$. Let $\theta_{1}=\frac{\pi}{2}$. Then

$$
0<k(\theta) \leq k\left(\theta_{1}\right)=\frac{1}{2}
$$

and it follows from $(2.12),(2.20)$ and $(2.21)$ that

$$
\begin{aligned}
\pi>\operatorname{argh}\left(e^{i \theta}\right) & \geq \operatorname{argh}\left(e^{i \theta_{1}}\right)=\frac{1}{2}(b+1) \alpha \pi+\tan ^{-1}\left(\frac{\alpha}{\mu}\right) \\
& =\frac{\pi}{2} \beta>0 .
\end{aligned}
$$

(ii) If $k(\theta)<0$, then it follows from $(2.17)$ to $(2.19)$ that

$$
h\left(e^{i \theta}\right)=\left(-\cot \frac{\theta}{2}\right)^{(b+1) \alpha} e^{-\frac{1}{2}(b+1) \alpha \pi i}\left(\mu+i \frac{\alpha}{2}\left(\cot \frac{\theta}{2}+\tan \frac{\theta}{2}\right)\right),
$$

and so

$$
\operatorname{argh}\left(e^{i \theta}\right)=-\frac{1}{2}(b+1) \alpha \pi+\tan ^{-1}\left(\frac{\alpha}{2 \mu k(\theta)}\right)
$$

for $\mu>0, e^{i \theta} \neq 1$ and $e^{i \theta} \neq-1$. Let $\theta_{2}=-\frac{\pi}{2}$. Then

$$
0>k(\theta) \geq k\left(\theta_{2}\right)=-\frac{1}{2}
$$

and from (2.12), (2.23) and (2.24) we have

$$
\begin{aligned}
-\pi<\operatorname{argh}\left(e^{i \theta}\right) & \leq \operatorname{argh}\left(e^{i \theta_{2}}\right)=-\frac{1}{2}(b+1) \alpha \pi-\tan ^{-1}\left(\frac{\alpha}{\mu}\right) \\
& =-\frac{\pi}{2} \beta<0 .
\end{aligned}
$$

Noting that $h(0)=\mu>0$, we find from (2.22) and (2.25) that $h(U)$ properly contains the angular region $-\frac{\pi}{2} \beta<\arg w<\frac{\pi}{2} \beta$ in the complex $w$-plane. Consequently, if $f(z) \in A_{p}$ satisfies (2.13), then the subordination relation (2.16) holds true, and so we have the assertion (2.15) of Theorem 3.

Furthermore, for the function $f(z) \in A_{p}$ defined by (2.4), we have (2.15) and

$$
\mu\left(\frac{z\left(Q_{p, \lambda_{2}}^{\lambda_{1}} f(z)\right)^{\prime}}{p Q_{p, \lambda_{2}}^{\lambda_{1}} f(z)}\right)^{b+1}+z\left(\frac{z\left(Q_{p, \lambda_{2}}^{\lambda_{1}} f(z)\right)^{\prime}}{p Q_{p, \lambda_{2}}^{\lambda_{1}} f(z)}\right)^{\prime}\left(\frac{z\left(Q_{p, \lambda_{2}}^{\lambda_{1}} f(z)\right)^{\prime}}{p Q_{p, \lambda_{2}}^{\lambda_{1}} f(z)}\right)^{b}=h(z) .
$$

Hence, by using (2.22) and (2.25), we conclude that the bound $\beta$ in (2.13) is the best possible. This completes our proof.

Acknowledgement. I would like to express sincere thanks to the referee for careful reading and suggestions which helped me to improve the paper. 


\section{References}

[1] M. K. Aouf, H. M. Hossen, and A. Y. Lashin, An application of certain integral operators, J. Math. Anal. Appl. 248 (2000), no. 2, 475-481.

[2] I. B. Jung, Y. C. Kim, and H. M. Srivastava, The Hardy space of analytic functions associated with certain one-parameter families of integral operators, J. Math. Anal. Appl. 176 (1993), no. 1, 138-147.

[3] J.-L. Liu, Notes on Jung-Kim-Srivastava integral operator, J. Math. Anal. Appl. 294 (2004), no. 1, 96-103.

[4] J.-L. Liu and S. Owa, Properties of certain integral operator, Int. J. Math. Sci. 3 (2004), no. 2, 351-359.

[5] S. S. Miller and P. T. Mocanu, Differential Subordinations: Theory and Applications, Series in Pure and Applied Mathematics, No. 225, Marcel Dekker, New York, 2000.

DePARTMENT OF Mathematics

YANGZHOU UNIVERSITY

Yangzhou 225002, P. R. China

E-mail address: jlliu@yzu.edu.cn 